\title{
Formación de médicos en postgrado de ginecología mediante entrenamiento simulado
}

Analia Grenno Troitiño1, Diego Greif Waldman¹,Fernanda Gómez Castro1, Soledad Bottaro Carve ${ }^{1}$, María Fernanda Nozar Cabrera², Verónica Fiol Lepera², Francisco Cóppola Gonzalvez ${ }^{3}$, Leonel Briozzo Colombo ${ }^{4}$

\section{RESUMEN}

Objetivo: Presentar la primera experiencia en nuestro medio, en simulación para formación de Residentes de Ginecología de la Facultad de Medicina de Montevideo, Universidad de la República.

Material y Métodos: La actividad docente se dividió en IV Módulos: I- Seguridad del Paciente y Humanismo médico, IIAnticoncepción, III- Parto instrumental y Sutura de episiotomía y IV - Emergencias obstétricas. Se realizaron exposiciones teóricas e instancias prácticas con simuladores inertes, de baja y moderada complejidad y simulación de situaciones clínicas. Se realizó autoevaluación, evaluación entre pares, aprendizaje colaborativo. Pre test y post test de conocimientos. Resultados: Los Residentes concuerdan que es una práctica muy útil y destacaron la oportunidad que la simulación brinda para practicar sin riesgo, entrenar el trabajo en equipo y actualizar guías clínicas sobre situaciones críticas.

Conclusión: Ésta fue una iniciativa muy favorable para el desarrollo del área de educación médica, ya que propone una metodología de aprendizaje y de evaluación objetiva, así como un espacio para que los estudiantes puedan desarrollar destrezas y habilidades, enfrentándose con una mayor seguridad y profesionalismo al paciente. (Horiz Med 2014; 14(3): 6-11)

Palabras clave: simulación clínica, paciente simulado, seguridad del paciente. (Fuente: DeCS BIREME).

\section{Training of postgraduate doctors in gynecology using simulators}

\begin{abstract}
Objective: To present the first experience with simulation for Ob/Gyn Residents in our medical community, in Montevideo School of Medicine, University of the Republic (UdeLaR).

Material and Methods: The educational program was divided into 4 modules: I- Patient safety and medical humanism, IIContraception, III- Assisted birth and episiotomy suturing and IV- Obstetric emergencies. Theoretical expositions, practical experiences with inert simulators of higher and lower complexity and simulation of clinical situations were performed. The students carried out instances of auto evaluation, peer evaluation, and collaborative learning. Also took pre- and post- tests to assess knowledge.

Results: The Residents agreed in the usefulness of this practice, and highlighted the advantages simulators provide: a riskfree learning environment, an opportunity for exercising team work and updating clinical guidelines in critical conditions. Conclusion: This was a very favorable development in our medical education department, since it provided a learning and objective evaluating methodology, as well as an environment that allowed students to develop skills and abilities, and to approach patients with more professionalism and confidence. (Horiz Med 2014; 14(3): 6-11)
\end{abstract}

Key words: Patient simulation, clinic simulation, patient safety. (Source: MeSH NLM).

Asistente de Clínica Ginecotocológica “A” Centro Hospitalario Pereira Rossell. Facultad de Medicina. Universidad de la República Uruguay

2 Profesor Adjunto Clínica Ginecotocológica “A” Centro Hospitalario Pereira Rossell. Facultad de Medicina. Universidad de la República. Uruguay

3 Profesor Agregado Clínica Ginecotocológica “A” Centro Hospitalario Pereira Rossell. Facultad de Medicina. Universidad de la República. Uruguay

4 Profesor Clínica Ginecotocológica “A” Centro Hospitalario Pereira Rossell. Facultad de Medicina. Universidad de la República. Uruguay 


\section{INTRODUCCIÓN}

En Uruguay, para ingresar a la carrera de especialista en Ginecotocología, el médico debe demostrar competencias, habilidades, destrezas y conocimientos sobre la especialidad elegida, mediante una prueba teórica. Asumir que han adquirido estas habilidades durante su formación como estudiantes de grado podría resultar incorrecto. Las habilidades comunicacionales, destrezas técnicas y no técnicas integran las competencias esenciales que deben alcanzar todos los Residentes de Ginecotocología en su formación como especialista.

El problema es, que al comenzar el primer año de su formación como postgrados, con muy poca experiencia, ya se ven enfrentados diariamente a situaciones de urgencia, emergencia y a diversos procedimientos técnicos gineco-obstétricos directamente en el ambiente asistencial de nuestros servicios, por lo que su entrenamiento en esta etapa es fundamental.

Para intentar solucionar éste problema, se ha incluido recientemente en la currícula de los Residentes de primer año de la Clínica Ginecotocológica "A" de la Facultad de Medicina de Montevideo de la Universidad de la República Oriental del Uruguay (UDELAR) la experiencia en el Laboratorio de Aprendizaje. En él, se puede aprender y practicar distintas técnicas con objetos inanimados (simuladores-maniquíes ginecológicos y obstétricos). Figura 1.

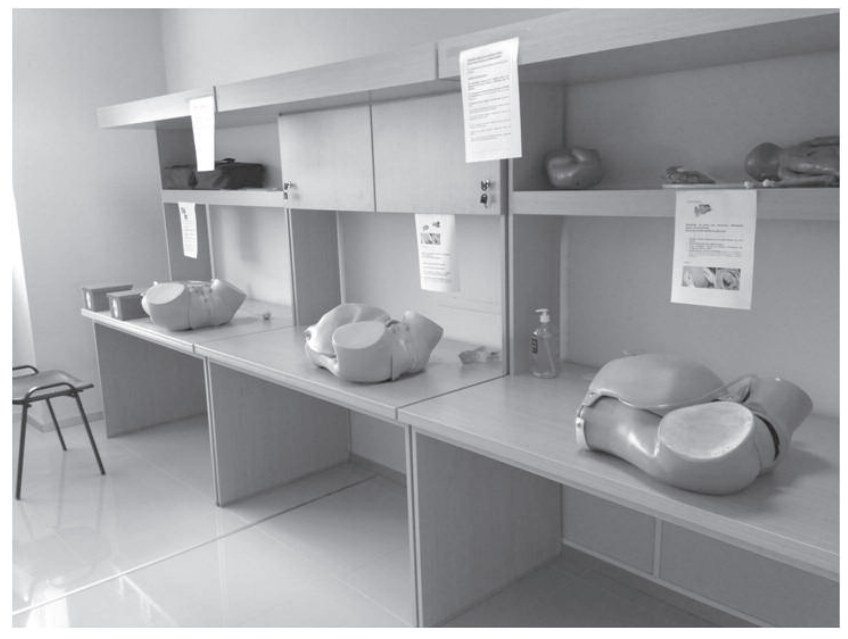

Figura 1. Laboratorio de aprendizaje
La simulación en ginecología y obstetricia se ha desarrollado gracias al uso de simuladores de habilidades específicas, modelos humanos básicos y avanzados, realidad táctil y escenarios simulados (1). Mediante su manejo, se pueden trabajar habilidades técnicas y no técnicas que permiten una experiencia de aprendizaje muy próxima a la realidad.

El uso de simuladores mejora las habilidades y el desempeño cuando nos enfrentamos a situaciones reales (2). La simulación clínica es un método seguro de aprendizaje centrado en el estudiante (3), permitiendo una experiencia que replica la realidad en un entorno seguro sin poner en riesgo a los pacientes y a los aprendices $(3,4)$.

Otro aspecto importante de ésta metodología, es que permite la evaluación formativa de los Residentes. La autoevaluación o feedback (devolución constructiva del desempeño ante una situación clínica real o simulada), así como evaluación entre pares, "Residente como profesor" juegan un papel muy importante en la enseñanza (5). Los Residentes necesitan ser capacitados formalmente para lograr ésta retroalimentación y evaluación ya que forma parte de su evaluación formativa y se asocia claramente con una enseñanza de alta calidad (6).

En Uruguay, en la formación del postgrado de ginecología, aun predomina la adquisición de habilidades y destrezas directamente en el proceso asistencial de pacientes que se asisten en el servicio de asistencia pública, donde acude la población más vulnerable desde el punto de vista sanitario, económico y cultural. En el corriente año, se está comenzando con experiencias aisladas de formación de ginecólogos en el ámbito privado de la asistencia sanitaria del país.

En ésta publicación, se presenta la primera experiencia en Simulación con Residentes de Ginecología de la Facultad de Medicina de Montevideo.El objetivo de las Jornadas es que los Residentes adquieran habilidades, destrezas y competencias en diferentes procedimientos gineco-obstétricos, que les permitirá un mejor desempeño en la práctica clínica. 


\section{MATERIAL Y MÉTODOS}

Las Jornadas fueron realizadas en mayo de 2014 y tuvieron una duración total de 20 horas, divididas en 2 días. Las exposiciones sobre conocimientos teóricos tuvieron una duración de 6 horas, mientras que las actividades prácticas una duración de 14 horas. Dichas jornadas, fueron destinadas a Residentes de primer año de ginecología y optativamente para los de segundo y tercer año.

Participaron un total de 17 Residentes. Las diferentes actividades fueron elaboradas y coordinadas por Docentes de la Clínica Ginecotocológica "A" de la Facultad de Medicina.

Los contenidos se dividieron en IV Módulos:

I - Seguridad del Paciente y Humanismo médico

II - Anticoncepción

III- Parto instrumental y Sutura de episiotomía

IV- Emergencias obstétricas.

En el módulo I, se realizaron dos exposiciones de conocimientos teóricos de 20 minutos de duración, sobre Seguridad del paciente y Humanismo Médico. Posteriormente, se realizó una actividad de discusión de situaciones habituales en la práctica clínica por parte de los estudiantes, con un proceso de reflexión sobre los mismos.

El módulo II, comenzó con una exposición sobre criterios médicos de elegibilidad para el uso de anticonceptivos. Luego previo a la instancia práctica, se realizó una exposición sobre conocimientos teóricos, aspectos técnicos y anatómicos, sobre colocación de dispositivo intrauterino (DIU) y colocación de implantes subdérmicos. Se observaron videos explicativos de los diferentes procedimientos (los cuales fueron elaborados previamente por los docentes coordinadores).

Para la práctica de habilidades y destrezas para la inserción y extracción de Dispositivos intrauterinos ( $T$ de Cobre), se utilizó simuladores de pelvis femenina de tamaño normal, maniquíes inertes, no interactivos de baja complejidad (ZOE $®$ S 504.100).
Para la colocación de Implantes subdérmicos se utilizó simuladores inertes de polifón de miembro superior y se observó la colocación de los implantes in vivo a 3 mujeres, por parte del equipo docente. (Figura 2)

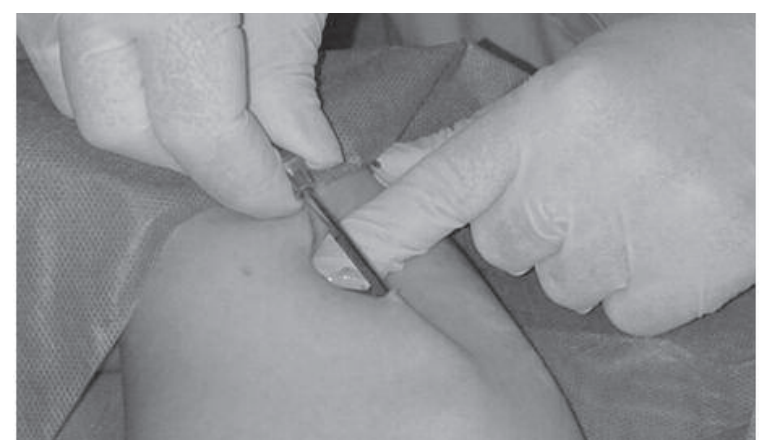

Figura 2. Colocación de implantes subdérmicos

Las instancias prácticas fueron realizadas por todos los Residentes, y presenciadas por los docentes coordinadores, lo que permitió la retroalimentación, la autoevaluación, evaluación entre pares, así como el aprendizaje colaborativo (7).

El módulo III, sobre Parto instrumental y Sutura de episiotomía, al igual que el módulo anterior se realizó una exposición sobre conocimientos teóricos, aspectos técnicos y anatómicos previos a la realización de las actividades prácticas.

Para la práctica de parto instrumental, se utilizó simuladores S500, Simulador Avanzado de Parto y simulador obstétrico (Simularis $®$ ). Se utilizaron tres tipos de fórceps: Fórceps Simpson, Fórceps Tarnier y Espátulas de Thierry. (Figura 3)

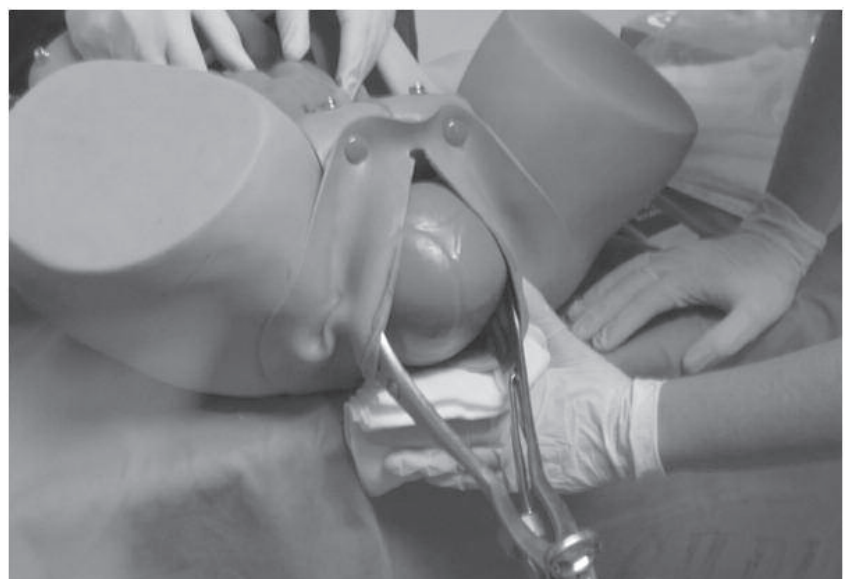

Figura 3. Aplicación de fórceps 
Las habilidades y destrezas en la técnica de sutura de episiotomía, se realizó en modelos de polifón, elaborados por los docentes. Se utilizaron porta agujas, pinzas de disección, tijeras de Mayo e hilos de sutura poliglactina 910.(Figura 4).

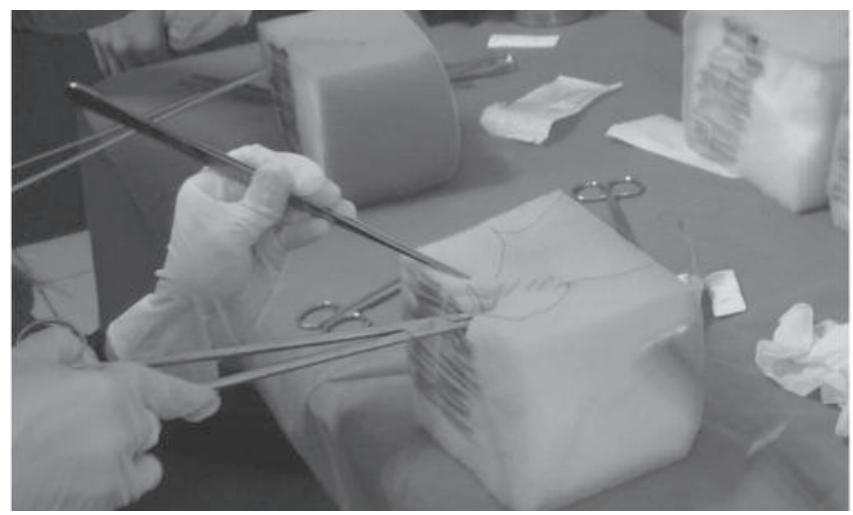

Figura 4. Sutura de episiotomía

En el módulo IV, se realizaron situaciones clínicas simuladas (emergencias obstétricas). Se seleccionaron cinco situaciones a las cuales los profesionales deben estar familiarizados: Distocia de hombro, Procidencia de cordón, Hipoxia fetal intraparto, Eclampsia y Hemorragia del Alumbramiento. En cada una de ellas se elaboró un guión, simulando una situación clínica real, en concordancia con los objetivos de aprendizaje establecidos.

Se utilizó la metodología de paciente simulado (8), y se contó con la participación de actrices profesionales de la Escuela Municipal de Arte Dramático (simulaban ser pacientes reales). Los docentes simulaban ser parte del equipo de salud (enfermeras/os) y los Residentes ejercían su rol real. Éstos últimos, no conocían previamente las situaciones clínicas a las que se iban a enfrentar, se le explicaba previamente que iban a participar de una simulación y que debían de actuar tal cual lo haría en la vida real.

Para la Simulación de Hipoxia fetal intraparto se utilizó un simulador de mediana complejidad, interactivo NOELLE \& S 552, Gaumard. Para la simulación de distocia de hombros y la procidencia de cordón se utilizaron los simuladores $\mathrm{S} 500$ y la adaptación S500.3 Simulador Avanzado de Parto y simulador obstétrico (Simularis ${ }^{\circledR}$ ). A su vez, en la simulación de distocia de hombros se utilizó la participación de actores. Para la simulación de la eclampsia: participaron actores y set de módulos de dilatación y borramiento cervical. Para la simulación de la hemorragia puerperal se utilizaron actores reales, mediante la dramatización para el caso clínico, y simuladores en el examen físico (ZOE (B) S 504.100).Figura 5.

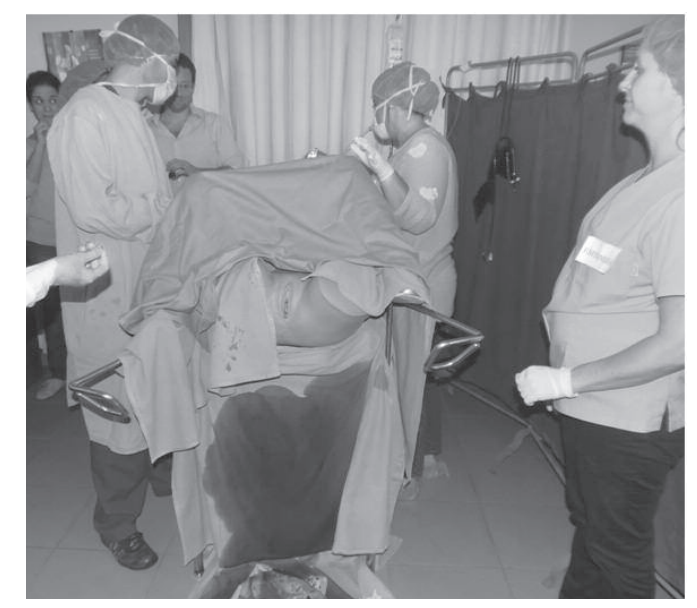

Figura 5. Simulación emergencias obstétricas. Hemorragia puerperal

Se utilizó un sistema de grabación audiovisual, con transmisión en tiempo real al auditorio, para los Residentes que no participaban de la simulación. Durante la dramatización los estudiantes del auditorio utilizaban una lista de cotejos para el análisis de la situación clínica. Esto permitió el feedback entre pares y por los docentes. Luego de la evaluación de cada dramatización, se realizó una breve exposición teórica, resumiendo aspectos relevantes del manejo clínico recomendado por las guías de práctica clínica.

\section{RESULTADOS}

Con respecto a la adquisición de conocimientos a lo largo del curso se destaca un $68,23 \%$ de respuestas correctas a nivel del pre test y del $92,2 \%$ en el postest.

Al final de la Jornadas, se solicitó a todos los Residentes que respondieran preguntas sobre satisfacción de la simulación como metodología de aprendizaje y la utilidad del Laboratorio de Aprendizaje. 
Analia Grenno Troitiño, Diego Greif Waldman,Fernanda Gómez Castro, Soledad Bottaro Carve, María

Fernanda Nozar Cabrera, Verónica Fiol Lepera, Francisco Cóppola Gonzalvez, Leonel Briozzo Colombo

Las encuestas fueron anónimas y se realizaron al total de los participantes. Los Residentes concuerdan en que es una práctica muy útil y destacaron la oportunidad que la simulación brinda para practicar sin riesgo, entrenar el trabajo en equipo y actualizar guías clínicas sobre situaciones estresantes. (Tabla 1).

Tabla 1. Características clínicas y Demográficas de la población estudiada

\begin{tabular}{|c|c|c|c|c|}
\hline En relación a esta jornada consideras que: & $\begin{array}{c}\text { Muy } \\
\text { adecuado }\end{array}$ & Adecuado & $\begin{array}{l}\text { Más o } \\
\text { menos }\end{array}$ & Nada \\
\hline La organización de la jornada fue & $100 \%$ & & & \\
\hline El tiempo dedicado fue & $94,20 \%$ & $5,80 \%$ & & \\
\hline El respeto a los horarios & $82,40 \%$ & $17,60 \%$ & & \\
\hline El nivel de los contenidos teóricos abordados fue & $94,20 \%$ & $5,80 \%$ & & \\
\hline La utilidad de los videos didácticos fue & $94,20 \%$ & $5,80 \%$ & & \\
\hline La utilidad de las presentaciones teóricas fue & $100 \%$ & & & \\
\hline La utilidad de las situaciones simuladas fue & $100 \%$ & & & \\
\hline La capacidad docente fue & $100 \%$ & & & \\
\hline La jornada te permitió reflexionar sobre tu práctica diaria en un nivel & $100 \%$ & & & \\
\hline En líneas generales la jornada te ha parecido & $100 \%$ & & & \\
\hline
\end{tabular}

\section{DISCUSIÓN}

La simulación es una metodología de enseñanza y evaluación aplicada desde finales de los años 60 , en diferentes universidades de todo el mundo, con su auge en los años 80(8). Esta herramienta es muy útil en diferentes profesiones y sin lugar a dudas muy valiosa en las ciencias de la salud. Los anestesistas, cardiólogos e intensivistas tienen gran experiencia al respecto y el uso en ginecología y obstetricia ha ido en aumento $(8,9)$.

La incorporación de ésta metodología de aprendizaje no intenta de ningún modo sustituir la práctica clínica con el paciente real, tan solo es un recurso complementario para la formación y el entrenamiento de destrezas (10). Su utilidad parece ser cada vez de mayor importancia, especialmente en aquellas situaciones clínicas poco frecuentes y de muy alto riesgo (11).

La falta que existe hoy en día en la currícula de los estudiantes de postgrado en formación en habilidades y destrezas previo al contacto con el paciente es una realidad. Gracias a que en los últimos años en nuestros ámbitos se está intentando innovar en metodologías de aprendizaje utilizando la simulación y los laboratorios de aprendizaje, éste problema posiblemente se pueda solucionar.
La necesidad de preservar la seguridad del paciente, en el proceso de aprendizaje del estudiante, ha hecho que la simulación sea un imperativo ético. La simulación en gineco-obstetricia puede disminuir el conflicto ético del aprendizaje con el paciente ya que preserva su seguridad e intimidad.

Para que se logre la enseñanza mediante la simulación es que la Clínica Ginecotocológica "A" de la Facultad de Medicina, ha creado un Laboratorio de Aprendizaje y llevó a cabo estas Jornadas para Residentes. Éste es un proyecto educativo que pretende favorecer la adquisición de competencias, habilidades, destrezas técnicas y no técnicas.

El uso de simuladores o de situaciones clínicas simuladas (rollplaying, paciente simulado) requiere de una elaboración meticulosa, usando determinadas reglas, que deben de cumplirse (12). Según el tipo de simulador utilizado y de la situación real que se pretenda reproducir, se requiere previamente saber cuáles son los objetivos que se pretende alcanzar, mostrar las fortalezas y debilidades de la actividad. Cuanto más complejas sean las situaciones que se pretendan reproducir, como las emergencias obstétricas, más importante es definir los roles de los participantes.

En el caso de la utilización de actores (profesionales o no, pacientes voluntarios) es importante que éstos 
sean entrenados adecuadamente. Es así que la Facultad de Medicina tiene ya un camino transitado junto a la Escuela Municipal de Arte Dramático de Montevideo, en el entrenamiento de actores en la simulación de situaciones clínicas, que participan en la formación de estudiantes de grado.

La simulación permite evaluar competencias transversales de la práctica clínica, habilidades comunicacionales, profesionalismo, ética, liderazgo, trabajo individual y en equipo (8).

La simulación de situaciones reales permite también destacar aspectos no técnicos como: en el liderazgo, compromiso, compañerismo, profesionalismo, ética médica, empatía etc., no menos importantes que los estrictamente técnicos (conocimientos teóricos, habilidades y destrezas prácticas). El aprendizaje colaborativo entre pares es muy enriquecedor y les permite ascender en la pirámide de competencias clínicas, mejorando la eficacia del aprendizaje (13).

En conclusión, creemos que ésta fue una iniciativa muy favorable para el desarrollo del área de educación médica, ya que propone una metodología de aprendizaje y de evaluación objetiva, así como un espacio para que los estudiantes puedan desarrollar destrezas y habilidades, enfrentándose con una mayor seguridad y profesionalismo al paciente.

El uso de situaciones clínicas simuladas puede, sin lugar a dudas, ser de gran ayuda a la hora de adquirir experiencia en situaciones obstétricas poco frecuentes pero de alto riesgo, que requieren de una resolución pronta y expedita para disminuir las complicaciones materno-fetales.

Creemos que debería incluirse en forma obligatoria en el plan de estudio de los Postgrados y Residentes de Ginecotocología de nuestro país, y así mejorar la calidad de la educación y la atención médica.

\section{Fuentes de financiamiento}

Autofinanciado por los autores.

\section{Conflictos de interés}

Los autores declaran no tener ningún conflicto de interés.

\section{REFERENCIAS BIBLIOGRÁFICAS}

1. Rodríguez M.C, Díez N, Beunza JJ, Auba M, Olartecoechea B, RuizZambrana A, et al. Confianza de los estudiantes de medicina en el aprendizaje de la exploración obstétrica con simuladores.An Sist Sanit Navar 2013; 36(2):275-80.

2. Baeza MA, Mella J, Soldati A, Escudero E, Tapia A, Arraztoa JA. Aplicabilidad de simuladores de mediana complejidad en el proceso de formación de residentes de Ginecología y Obstetricia. Rev Chil Obstet Ginecol 2010; 75(6): 349 -354.

3. Ker J, Bradley P. Simulation in medical education. The Association for the Study of Medical Education 2010; 1-17.

4. Hernandez P, et al. Entrenamiento de equipos interdisciplinares en urgencias obstétricas mediante simulación clínica. Prog Obstet Ginecol 2011; 54(12):618-624.

5. Ricciotti HA, Dodge LE, Head J, Atkins M, Hacker MR. A novel resident-as-teacher training program to improve and evaluate obstetrics and gynecology residentte a chings kills. OBGYN Resident-as-Teacher Program 2012; 34: e52-e57

6. Alves de Lima A, E. Devolución constructiva una estrategia para mejorar el aprendizaje. Instituto Cardiovascular de Buenos Aires. Medicina 2008; 68(1).

7. Palacios $M$. Uso de pacientes estandarizados en educación médica. Rev Educ Cienc Salud 2007; 4 (2): 102-105.

8. Reynolds A,et al, Utilização das metodologias de simulação médica. No Ensino e Aprendizagem nas Escolas Médicas Portuguesas, Acta Med Port 2011; 24(2):223-230.

9. Gardner R, Raemer DB. Simulation in obstetrics and gynecology. Obstet Gynecol Clin North Am 2008;35:97-127.

10. Amaya AA. Simulación clínica:ipretende la educación médica basada en la simulación remplazar la formación tradicional en medicina y otras ciencias de la salud en cuanto a la experiencia actual con los pacientes? Univ. Méd. Bogotá (Colombia), 49(3): 399-405, [actualizadojul-sep 2008].

11. Goff B, Lentz G, Lee D, Mandel L. Formal Teaching of Surgical Skills in an Obstetric-Gynecologic Residency. Obstet Gynecol 1999;93:785-90.

12. Vázquez-Mata G; Guillamet-Lloveras A. El entrenamiento basado en la simulación como innovación imprescindible en la formación médica. Educ Med 2009; 12 (3): 149-155.

13. Durante E.La evaluación de los conocimientos: Lo que parece ser, ¿es realmente lo que es?.Rev Hospltal B.Aires 2005; 25(1):18-23.

\section{Correspondencia:}

Diego Greif

Dirección: Osorio 1321/703 Montevideo, Uruguay.

Teléfono: + 59899288990

Correo electrónico: diegogreif@gmail.com

Recibido: 07 de Julio de 2014

Aprobado: 05 de Setiembre de 2014 